\title{
Stationary Patterns of a Cross-Diffusion Epidemic Model
}

\author{
Yongli Cai, ${ }^{1,2}$ Dongxuan Chi, ${ }^{3}$ Wenbin Liu, ${ }^{4}$ and Weiming Wang ${ }^{2}$ \\ ${ }^{1}$ School of Mathematics and Computational Science, Sun Yat-sen University, Guangzhou 510275, China \\ ${ }^{2}$ College of Mathematics and Information Science, Wenzhou University, Wenzhou 325035, China \\ ${ }^{3}$ Department of Applied Mathematics, Shanghai Finance University, Shanghai 201209, China \\ ${ }^{4}$ College of Physics and Electronic Information Engineering, Wenzhou University, Wenzhou 325035, China
}

Correspondence should be addressed to Yongli Cai; caiyongli06@163.com

Received 16 September 2013; Accepted 3 October 2013

Academic Editor: Carlo Bianca

Copyright (C) 2013 Yongli Cai et al. This is an open access article distributed under the Creative Commons Attribution License, which permits unrestricted use, distribution, and reproduction in any medium, provided the original work is properly cited.

We investigate the complex dynamics of cross-diffusion SI epidemic model. We first give the conditions of the local and global stability of the nonnegative constant steady states, which indicates that the basic reproduction number determines whether there is an endemic outbreak or not. Furthermore, we prove the existence and nonexistence of the positive nonconstant steady states, which guarantees the existence of the stationary patterns.

\section{Introduction}

In epidemiology, epidemic compartmental models, since the pioneer work of Kermack and McKendrick [1], are widely used for increasing the understanding of infectious disease dynamics and for determining preventive measures to control infection spread qualitatively and quantitatively [2]. More recently, many studies have shown that a spatial epidemic model is an appropriate tool for investigating the fundamental mechanism of complex spatiotemporal epidemic dynamics. In these studies, reaction-diffusion equations have been intensively used to describe spatiotemporal dynamics [3-21]. Spatial epidemiology with diffusion has become a principal scientific discipline aiming at understanding the causes and consequences of spatial heterogeneity in disease transmission [20].

In addition, from a biological perspective, the diffusion of individuals may be connected with other things, such as searching for food, escaping high infection risks. In the first case, individuals tend to diffuse in the direction of lower density of a population, where there are richer resources. In the second, individuals may move along the gradient of infectious individuals to avoid higher infection [12, 22]. Keeping these in view, cross-diffusion arises, which was proposed first by Kerner [23] and first applied in competitive population system by Shigesada et al. [24]. In particular, Sun et al. [16], by using the standard linear analysis, studied the pattern formation in a cross-diffusion SI epidemic model. And in [19], the authors presented Turing pattern selection in a crossdiffusion SI epidemic model with zero-flux boundary conditions, gave the conditions of Hopf and Turing bifurcations, and derived the amplitude equations for the excited modes.

In the past decades, it has been shown that the reactiondiffusion system is capable to generate complex spatiotemporal patterns, and the existence of stationary patterns induced by diffusion has attracted the extensive attention of a great number of biologists and mathematicians, and lots of fascinating and important phenomena have been observed [2533]. In particular, in the field of epidemiology, there are many contributions to the existence of steady states in the diffusive epidemic models [34-45]. But in the studies on the steady states of diffusive epidemic models, little attention has been paid to study on the effect of cross-diffusion.

The main focus of this paper is to investigate how crossdiffusion affects disease's dynamics through studying the existence of the constant and nonconstant steady states of a cross-diffusion SI epidemic model.

The rest of this paper is organized as follows. In Section 2, we derive a cross-diffusion SI epidemic model. In Section 3, we give the global existence and positivity of the solution. In Section 4, we study the local and global stability of the nonnegative steady states of the model. In Section 5, we first 
give a priori estimates for the positive solutions of the model and then give some results on the existence and nonexistence of positive nonconstant steady states of the model. The paper ends with a brief discussion in Section 6 .

\section{Model Derivation}

Assume that the habitat $\Omega \subset \mathbb{R}^{m}(m \geq 1)$ is a bounded domain with smooth boundary $\partial \Omega$ (when $m>1$ ), and $\mathbf{n}$ is the outward unit normal vector on $\partial \Omega$. We consider the following cross-diffusion SI epidemic model:

$$
\begin{gathered}
\frac{\partial S}{\partial t}-d_{S} \Delta S=r S\left(1-\frac{S}{K}\right)-\frac{\beta S I}{S+I}, \quad x \in \Omega, t>0, \\
\frac{\partial I}{\partial t}-D \Delta S-d_{I} \Delta I=\frac{\beta S I}{S+I}-\mu I, \quad x \in \Omega, t>0, \\
\frac{\partial S}{\partial \mathbf{n}}=\frac{\partial I}{\partial \mathbf{n}}=0, \quad x \in \partial \Omega, \\
S(x, 0)=S_{0}(x)>0, \quad I(x, 0)=I_{0}(x) \geq 0, \quad x \in \Omega,
\end{gathered}
$$

where $S(x, t)$ and $I(x, t)$ denote the density of susceptible and infected individuals at location $x \in \Omega$ and time $t$, respectively, $d_{S}$ and $d_{I}$ are the self-diffusion coefficients for the susceptible and infected individuals, and $D$ is the cross-diffusion coefficient. $r$ stands for the susceptible population intrinsic growth rate, $\beta$ the rate of transmission, $\mu$ the death rate of the infected population $I$, and $K$ the carrying capacity. The symbol $\Delta$ is the Laplacian operator. The homogeneous Neumann boundary condition implies that the above model is self-contained and there is no infection across the boundary.

It is worthy to note that the diffusion coefficients $d_{S}, d_{I}$, and $D$ are such that $d_{S}, d_{I}>0, D \geq 0$ and $d_{S}>d_{I}, D^{2}<$ $4 d_{S} d_{I}$ which is the parabolic condition.

For model (1), the basic reproduction number is defined as

$$
R_{0}=\frac{\beta}{\mu} .
$$

The steady states of model (1) satisfy

$$
\begin{gathered}
-d_{S} \Delta S=r S\left(1-\frac{S}{K}\right)-\frac{\beta S I}{S+I}, \quad x \in \Omega, \\
-D \Delta S-d_{I} \Delta I=\frac{\beta S I}{S+I}-\mu I, \quad x \in \Omega, \\
\frac{\partial S}{\partial \mathbf{n}}=\frac{\partial I}{\partial \mathbf{n}}=0, \quad x \in \partial \Omega, t>0 .
\end{gathered}
$$

Throughout this paper, the positive solution $(S, I)$ satisfying (1) refers to a classical one with $S>0, I>0$ on $\bar{\Omega}$. Clearly, model (1) has a semitrivial constant solution (diseasefree equilibrium) $E_{0}=(K, 0)$ and a positive constant solution (endemic equilibrium)

$$
\begin{aligned}
E^{*} & =\left(S^{*}, I^{*}\right) \\
& =\left(\frac{K}{r}\left(r-\left(R_{0}-1\right) \mu\right), \frac{K}{r}\left(r-\left(R_{0}-1\right) \mu\right)\left(R_{0}-1\right)\right)
\end{aligned}
$$

if $1<R_{0}<1+(r / \mu)$.

\section{Global Existence and Positivity of the Solution}

In this section, we show the existence of unique positive global solution of model (1).

First, we convert model (1) into an abstract first-order system $C(\bar{\Omega}) \times C(\bar{\Omega})$ of the form

$$
\begin{gathered}
U^{\prime}(t)=A U(t)+F(U(t)), \quad t>0, \\
U(0)=U_{0} \in C(\bar{\Omega}) \times C(\bar{\Omega}),
\end{gathered}
$$

where

$$
\begin{gathered}
A U(t)=\left(d_{S} \Delta S, D \Delta S+d_{I} \Delta I\right), \\
F(U(t))=\left(r S\left(1-\frac{S}{K}\right)-\frac{\beta S I}{S+I}, \frac{\beta S I}{S+I}-\mu I\right) .
\end{gathered}
$$

Since $F$ is locally Lipschitz in $C(\bar{\Omega}) \times C(\bar{\Omega})$, for every initial date $U_{0} \in C(\bar{\Omega}) \times C(\bar{\Omega})$, system (5) admits a unique local solution on $\left[0, T_{\max }\right)$, where $T_{\max }$ is the maximal existence time for solution of system (5) [46].

Set $Z=I-\left(D /\left(d_{S}-d_{I}\right)\right) S$. Then model (1) leads to

$$
\begin{gathered}
\frac{\partial S}{\partial t}-d_{S} \Delta S=r S\left(1-\frac{S}{K}\right) \\
-\frac{\beta S I}{S+I}:=\Lambda(S, Z), \quad x \in \Omega, t>0, \\
\frac{\partial Z}{\partial t}-d_{I} \Delta Z=\frac{D}{d_{S}-d_{I}}\left(r S\left(1-\frac{S}{K}\right)-\frac{\beta S I}{S+I}\right) \\
+\frac{\beta S I}{S+I}-\mu I:=\Upsilon(S, Z), \quad x \in \Omega, t>0, \\
\frac{\partial S}{\partial \mathbf{n}}=\frac{\partial Z}{\partial \mathbf{n}}=0, \quad x \in \partial \Omega, t>0, \\
S(x, 0)=S_{0}(x)>0, \\
Z(x, 0)=Z_{0}(x)=I_{0}(x)-\frac{D}{d_{S}-d_{I}} S_{0}(x), \quad x \in \Omega .
\end{gathered}
$$

A simple application of a comparison theorem to model (7) implies (see [47]) that for positive initial date $S_{0}(x)>0$ and $Z_{0}(x) \geq 0$ we have that

$$
S(x, t)>0, \quad I(x) \geq \frac{D}{d_{S}-d_{I}} S(x, t), \quad \forall x \in \Omega, t>0 .
$$

Applying the comparison principle we get that $S(x, t) \leq$ $\max \left\{\left\|S_{0}\right\|_{\infty}, K\right\}$. To establish the uniform boundedness of $I(x, t)$, it is sufficient to show the uniform boundedness of $Z(x, t)$. This task is carried out using a result found in Henry [48], from which it is sufficient to derive a uniform estimate for $\|\Upsilon(S, Z)\|_{p}$. Hence, we apply the same method of $[49,50]$ to study the existence of global solution of model (1). And we have the following result. 
Theorem 1. The solution of model (1) is global and uniformly bounded in $[0, \infty[$.

For the sake of simplicity, we omit the proof, and the interested readers may refer to $[49,50]$ for details.

\section{Stability of Nonnegative Constant Steady States}

In this section, we consider the stability behavior of nonnegative constant steady states to model (1).

4.1. Local Stability of Nonnegative Steady States. In this subsection, we will discuss the local stability of the constant steady states $E_{0}=(K, 0)$ and $E^{*}=\left(S^{*}, I^{*}\right)$. For this purpose, we need to introduce some notations.

Let $0=\lambda_{0}<\lambda_{1}<\lambda_{2}<\cdots$ be the eigenvalues of the operator- $\Delta$ on $\Omega$ with the homogeneous Neumann boundary conditions. Let $\mathbf{X}=\left\{(S, I) \in\left[C^{2}(\Omega)\right]^{2} \mid \partial S / \partial \mathbf{n}=\partial I / \partial \mathbf{n}=\right.$ $0, x \in \partial \Omega\},\left\{\phi_{i j} \mid j=1, \ldots, \operatorname{dim} E\left(\lambda_{i}\right)\right\}$ be an orthonormal basis of $E\left(\lambda_{i}\right)$, and $\mathbf{X}_{i j}=\left\{\mathbf{c} \phi_{i j} \mid \mathbf{c} \in \mathbb{R}^{2}\right\}$; then $\mathbf{X}=\bigoplus_{i=1}^{\infty} \mathbf{X}_{i}$ where $\mathbf{X}_{i}=\bigoplus_{j=1}^{\operatorname{dim} E\left(\lambda_{i}\right)} \mathbf{X}_{i j}$.

Theorem 2. For model (1),

(a) if $1<R_{0}<1+(r / \mu)$ and $d_{I}\left(\mu R_{0}^{2}-r R_{0}-\mu\right)+$ $D \mu\left(R_{0}-1\right)^{2}<d_{S} \mu\left(R_{0}-1\right)$, the positive constant steady state $E^{*}=\left(S^{*}, I^{*}\right)$ is locally asymptotically stable;

(b) if $R_{0} \leq 1$, the semitrivial constant steady state $E_{0}=$ $(K, 0)$ is locally asymptotically stable.

Proof. (a) The linearization of model (1) at the positive constant steady state $E^{*}=\left(S^{*}, I^{*}\right)$ can be expressed by

$$
\begin{gathered}
\frac{\partial \xi}{\partial t}=d_{S} \Delta \xi+\frac{\mu R_{0}^{2}-r R_{0}-\mu}{R_{0}} \xi-\frac{\mu}{R_{0}} \zeta, \quad x \in \Omega, t>0, \\
\frac{\partial \zeta}{\partial t}=D \Delta \xi+d_{I} \Delta \zeta+\frac{\mu\left(R_{0}-1\right)^{2}}{R_{0}} \xi \\
-\frac{\mu\left(R_{0}-1\right)}{R_{0}} \zeta, \quad x \in \Omega, t>0, \\
\frac{\partial \xi}{\partial \mathbf{n}}=\frac{\partial \zeta}{\partial \mathbf{n}}=0, \quad x \in \partial \Omega .
\end{gathered}
$$

Let

$$
\mathfrak{E}=\left(\begin{array}{cc}
d_{S} \Delta+\frac{\mu R_{0}^{2}-r R_{0}-\mu}{R_{0}} & -\frac{\mu}{R_{0}} \\
D \Delta+\frac{\mu\left(R_{0}-1\right)^{2}}{R_{0}} & d_{I} \Delta-\frac{\mu\left(R_{0}-1\right)}{R_{0}}
\end{array}\right) .
$$

For each $i \geq 0, \mathbf{X}_{i}$ is invariant under the operator $£$, and $\eta$ is an eigenvalue of $£$ if and only if $\eta$ is an eigenvalue of the matrix

$$
A_{i}=\left(\begin{array}{cc}
-d_{S} \lambda_{i}+\frac{\mu R_{0}^{2}-r R_{0}-\mu}{R_{0}} & -\frac{\mu}{R_{0}} \\
-D \lambda_{i}+\frac{\mu\left(R_{0}-1\right)^{2}}{R_{0}} & -d_{I} \lambda_{i}-\frac{\mu\left(R_{0}-1\right)}{R_{0}}
\end{array}\right),
$$

for some $i \geq 0$. Thus the stability of the positive constant steady state is reduced to consider the characteristic equation:

$$
\operatorname{det}\left(\eta I-A_{i}\right)=\eta^{2}-\operatorname{trace}\left(A_{i}\right) \eta+\operatorname{det}\left(A_{i}\right):=\varphi_{i}(\eta)
$$

where

$$
\begin{gathered}
\operatorname{trace}\left(A_{i}\right)=-\lambda_{i}\left(d_{S}+d_{I}\right)-\left(r-\mu\left(R_{0}-1\right)\right)<0, \\
\operatorname{det}\left(A_{i}\right)=d_{S} d_{I} \lambda_{i}^{2}+\frac{1}{R_{0}}\left(d_{S} \mu\left(R_{0}-1\right)-d_{I}\left(\mu R_{0}^{2}-r R_{0}-\mu\right)\right. \\
\left.-D \mu\left(R_{0}-1\right)^{2}\right) \lambda_{i} \\
+\frac{\mu\left(R_{0}-1\right)\left(r-\mu\left(R_{0}-1\right)\right)}{R_{0}} .
\end{gathered}
$$

It follows from $d_{I}\left(\mu R_{0}^{2}-r R_{0}-\mu\right)+D \mu\left(R_{0}-1\right)^{2}<d_{S} \mu\left(R_{0}-\right.$ 1) that $\operatorname{det}\left(A_{i}\right)>0$. Therefore, the eigenvalues of the matrix $A_{i}$ have negative real parts. It thus follows from the RouthHurwitz criterion that, for each $i \geq 0$, the two roots $\eta_{i 1}$ and $\eta_{i 2}$ of $\varphi_{i}(\eta)=0$ all have negative real parts

In the following, we prove that there exists $\kappa>0$ such that

$$
\Re\left\{\eta_{i 1}\right\}, \mathfrak{R}\left\{\eta_{i 2}\right\} \leq-\kappa
$$

Let $\eta=\lambda_{i} \xi$; then $\varphi_{i}(\eta)=\lambda_{i}^{2} \xi^{2}-\operatorname{tr}\left(A_{i}\right) \lambda_{i} \xi+\operatorname{det}\left(A_{i}\right):=\widetilde{\varphi}_{i}(\eta)$. Since $\lambda_{i} \rightarrow \infty$ as $i \rightarrow \infty$, it follows that

$$
\lim _{i \rightarrow \infty} \frac{\widetilde{\varphi}_{i}(\eta)}{\lambda_{i}^{2}}=\xi^{2}+\left(d_{S}+d_{I}\right) \xi+d_{S} d_{I}:=\widetilde{\varphi}(\xi)
$$

Clearly, $\tilde{\varphi}(\xi)=0$ has two negative roots: $-d_{S},-d_{I}$. Let $d=$ $\min \left\{d_{S}, d_{I}\right\}$. By continuity, we see that there exists $i_{0}$ such that the two roots $\xi_{i 1}, \xi_{i 2}$ of $\widetilde{\varphi}_{i}(\eta)=0$ satisfy $\mathfrak{R}\left\{\xi_{i 1}\right\}, \mathfrak{R}\left\{\xi_{i 2}\right\} \leq$ $-(d / 2), \forall i \geq i_{0}$. In turn, $\mathfrak{R}\left\{\eta_{i 1}\right\}, \mathfrak{R}\left\{\eta_{i 2}\right\} \leq-\left(\lambda_{i} d / 2\right) \leq-(d / 2)$, $\forall i \geq i_{0}$. Let $-\widetilde{\kappa}=\max _{1 \leq i \leq i_{0}}\left\{\mathfrak{R}\left\{\eta_{i 1}\right\}, \mathfrak{R}\left\{\eta_{i 2}\right\}\right\}$. Then $\widetilde{\kappa}>0$ and (14) holds for $\kappa=\min \{\widetilde{\kappa}, d / 2\}$.

Consequently, the spectrum of $£$ which consists of eigenvalues lies in $\{\Re\{\eta\} \leq-\kappa\}$. In the sense of [48], we obtain that the positive constant steady state solution $E^{*}=\left(S^{*}, I^{*}\right)$ of model (1) is uniformly asymptotically stable.

(b) The stability of the semitrivial constant steady state $E_{0}=(K, 0)$ is reduced to consider the characteristic equation:

$$
\operatorname{det}\left(\eta I-A_{i}\right)=\eta^{2}-\operatorname{trace}\left(A_{i}\right) \eta+\operatorname{det}\left(A_{i}\right)
$$


where

$$
\begin{aligned}
& \operatorname{trace}\left(A_{i}\right) \\
& \quad=-\lambda_{i}\left(d_{S}+d_{I}\right)-\left(r+\mu\left(R_{0}-1\right)\right)<0 \\
& \operatorname{det}\left(A_{i}\right) \\
& \quad=d_{S} d_{I} \lambda_{i}^{2}+\left(d_{S} \mu\left(1-R_{0}\right)+d_{I} r\right) \lambda_{i}+r \mu\left(1-R_{0}\right)>0 .
\end{aligned}
$$

The remaining arguments are rather similar as above. The proof is complete.

4.2. Global Stability of the Nonnegative Steady States. This subsection is devoted to the global stability of $E_{0}=(K, 0)$ and $E^{*}=\left(S^{*}, I^{*}\right)$ for model (1).

First, we have the following lemma regarding the persistence property of the susceptible individuals which will play a critical role in the proof of the global stability of $E^{*}=\left(S^{*}, I^{*}\right)$.

Lemma 3. If $R_{0}<r / \mu, S(x, t)$ satisfies

$$
\liminf _{t \rightarrow \infty} \min _{\bar{\Omega}} S(x, t) \geq K\left(1-\frac{R_{0} \mu}{r}\right) .
$$

Proof. For all $t \geq 0, S(x, t)$ is an upper solution of the following problem:

$$
\begin{gathered}
\frac{\partial z}{\partial t}-d_{S} \Delta z=r z\left(1-\frac{\beta}{r}-\frac{z}{K}\right), \quad x \in \Omega, t>0, \\
\frac{\partial z}{\partial \mathbf{n}}=0, \quad x \in \partial \Omega, t>0, \\
z(x, 0)=S_{0}(x)>0, \quad x \in \bar{\Omega} .
\end{gathered}
$$

Let $S(t)$ be the unique positive solution of the problem

$$
\begin{gathered}
\frac{\partial w}{\partial t}=r w\left(1-\frac{\beta}{r}-\frac{w}{K}\right), \quad t>0, \\
w(0)=\max _{\bar{\Omega}} S_{0}(x)>0 .
\end{gathered}
$$

Then $S(t)$ is a lower solution of $(20)$. Since $\beta<r$ we have

$$
\lim _{t \rightarrow \infty} S(t)=K\left(1-\frac{\beta}{r}\right)=K\left(1-\frac{R_{0} \mu}{r}\right) .
$$

It follows by a comparison argument that

$$
\liminf _{t \rightarrow \infty} \min _{\bar{\Omega}} S(x, t) \geq K\left(1-\frac{R_{0} \mu}{r}\right) .
$$

The proof is complete.

Now, we give the result of the global stability of $E_{0}$ and $E^{*}$.

Theorem 4. For model (1),

(a) if $\mu<r$ and $1<R_{0}<(r+\mu) / 2 \mu$, then positive constant steady state $E^{*}=\left(S^{*}, I^{*}\right)$ of model (1) globally asymptotically stable; (b) if $R_{0} \leq 1$, the semitrivial constant steady state $E_{0}=$ $(K, 0)$ of model $(1)$ is globally asymptotically stable.

Proof. (a) We adopt the Lyapunov function:

$$
V(t)=\int_{\Omega}\left[V_{1}(S(x, t))+\nu V_{2}(I(x, t))\right] d x,
$$

where $V_{1}(S)=\int_{S^{*}}^{S}\left(\left(\xi-S^{*}\right) / \xi\right) d \xi, V_{2}(I)=v \int_{I^{*}}^{I}\left(\left(\eta-I^{*}\right) / \eta\right) d \eta$, and $v=S^{*} / I^{*}=1 /\left(R_{0}-1\right)$. Then $V(t) \geq 0$ and $V(t)=0$ if and only if $(S, I)=\left(S^{*}, I^{*}\right)$. Then,

$$
\begin{aligned}
\frac{d V}{d t}= & \int_{\Omega}\left(\frac{S-S^{*}}{S} \frac{\partial S}{\partial t}+\frac{\nu\left(I-I^{*}\right)}{I} \frac{\partial I}{\partial t}\right) d x \\
= & \int_{\Omega}\left(S-S^{*}\right)\left(r-\frac{r S}{K}-\frac{\beta I}{S+I}+d_{S} \frac{\Delta S}{S}\right) d x \\
& +\int_{\Omega} \nu\left(I-I^{*}\right)\left(\frac{\beta S}{S+I}-\mu+d_{I} \frac{\Delta I}{I}+D \frac{\Delta S}{I}\right) d x \\
= & \int_{\Omega} P(S, I) d x \\
& -\int_{\Omega}\left(\frac{d_{S} S^{*}}{S^{2}}|\nabla S|^{2}+\frac{d_{I} \nu I^{*}}{I^{2}}|\nabla I|^{2}+\frac{D \nu I^{*}}{I^{2}}|\nabla S||\nabla I|\right) d x,
\end{aligned}
$$

where

$$
\begin{aligned}
P(S, I)= & \left(S-S^{*}\right)\left(r-\frac{r S}{K}-\frac{\beta I}{S+I}\right) \\
& +\nu\left(I-I^{*}\right)\left(\frac{\beta S}{S+I}-\mu\right) .
\end{aligned}
$$

It follows from Lemma 3 that, for any $\varepsilon>0$, there exists $t_{0}>$ 0 , such that $S+I \geq S \geq K\left(1-\left(\mu R_{0} / r\right)\right)-\varepsilon$ for all $x \in \bar{\Omega}$ and $t \geq t_{0}$. And by some computational analysis, we have

$$
\begin{aligned}
P(S, I)= & \left(S-S^{*}\right)^{2}\left(-\frac{r}{K}+\frac{\beta I^{*}}{\left(S^{*}+I^{*}\right)(S+I)}\right) \\
& -\frac{\nu S^{*}}{\left(S^{*}+I^{*}\right)(S+I)}\left(I-I^{*}\right)^{2} \\
= & -\left(S-S^{*}\right)^{2}\left(\frac{r}{K}-\frac{\mu\left(R_{0}-1\right)}{S+I}\right) \\
& -\frac{1}{R_{0}\left(R_{0}-1\right)(S+I)}\left(I-I^{*}\right)^{2} \\
\leq & -\left(S-S^{*}\right)^{2}\left(\frac{r}{K}-\frac{\mu\left(R_{0}-1\right)}{K\left(1-\mu R_{0} / r\right)-\varepsilon}\right) \\
& -\frac{1}{R_{0}\left(R_{0}-1\right)(S+I)}\left(I-I^{*}\right)^{2} .
\end{aligned}
$$

Hence, in view of the conditions of the theorem and the arbitrariness of $\varepsilon$, we have $P(S, I) \leq 0$; that is, $d V / d t \leq 0$ for all $x \in \bar{\Omega}$ and $t \geq t_{0}$. So $V(t)$ decreases monotonically 
along a solution orbit and $E^{*}$ is globally asymptotically stable under the assumptions of the theorem.

(b) We adopt the Lyapunov function:

$$
V(t)=\int_{\Omega} I d x
$$

Then $V(t) \geq 0$ and $V(t)=0$ if and only if $I=0$. Then, if $R_{0} \leq 1$, we obtain

$$
\begin{aligned}
\frac{d V}{d t} & =\int_{\Omega} \frac{\partial I}{\partial t} d x \\
& =\int_{\Omega}\left(\frac{\beta S I}{S+I}-\mu I+d_{I} \Delta I+D \Delta S\right) d x \\
& \leq \int_{\Omega} \mu I\left(R_{0}-1\right) d x \leq 0 .
\end{aligned}
$$

It follows from $I=0$ and the second equation of model (1) that $S$ is a constant. As a consequence, from the first equation of model ( 1 ) and $S \neq 0$, we have $S=K$. Hence, $E_{0}=(K, 0)$ is globally asymptotically stable.

\section{Existence and Nonexistence of Positive Nonconstant Steady States}

In this section, we provide some sufficient conditions for the existence and nonexistence of nonconstant positive solution of model (3) by using the Leray-Schauder degree theory [51]. For the purpose, it is necessary to establish a priori positive upper and lower bounds for the positive solution of model (3).

5.1. A Priori Estimates. In order to obtain the desired bounds, we recall the following maximum principle [22] and Harnack inequality [52].

Lemma 5 (maximum principle, see [22]). Let $\Omega$ be a bounded Lipschitz domain in $\mathbb{R}^{m}$ and $g \in C(\bar{\Omega} \times \mathbb{R})$.

(a) Assume that $w \in C^{2}(\Omega) \cap C^{1}(\bar{\Omega})$ and satisfies

$$
\Delta w(x)+g(x, w(x)) \geq 0 \quad \text { in } \Omega, \quad \frac{\partial w}{\partial \mathbf{n}} \leq 0 \quad \text { on } \partial \Omega
$$

$$
\text { If } w\left(x_{0}\right)=\max _{\bar{\Omega}} w(x) \text {, then } g\left(x_{0}, w\left(x_{0}\right)\right) \geq 0 \text {. }
$$

(b) Assume that $w \in C^{2}(\Omega) \cap C^{1}(\bar{\Omega})$ and satisfies

$$
\Delta w(x)+g(x, w(x)) \leq 0 \quad \text { in } \Omega, \quad \frac{\partial w}{\partial \mathbf{n}} \geq 0 \quad \text { on } \partial \Omega .
$$

$$
\text { If } w\left(x_{0}\right)=\min _{\bar{\Omega}} w(x) \text {, then } g\left(x_{0}, w\left(x_{0}\right)\right) \leq 0 \text {. }
$$

Lemma 6 (Harnack inequality, see [52]). Let $w \in C^{2}(\Omega) \cap$ $C^{1}(\bar{\Omega})$ be a positive solution to $\Delta w(x)+c(x) w(x)=0$, where $c \in C(\bar{\Omega})$, satisfying the homogeneous Neumann boundary conditions. Then there exists a positive constant $C^{*}=C^{*}\left(\|c\|_{\infty}\right.$, $\Omega)$, such that

$$
\max _{\bar{\Omega}} w \leq C^{*} \min _{\bar{\Omega}} w
$$

For convenience, let us denote the constants $r, K, \beta$, and $\mu$ collectively by $\Lambda$. The positive constants $C, \underline{C}, \bar{C}$, and so forth will depend only on the domains $\Omega$ and $\Lambda$.

Theorem 7. Assume that $1<R_{0}<r / \mu$. Let $D_{1}$ be an arbitrary fixed positive number. Then there exist positive constants $\underline{C}=$ $\underline{C}\left(\Lambda, \Omega, D_{1}\right)$ and $\bar{C}=\bar{C}\left(\Lambda, \Omega, D_{1}\right)$, such that if $d_{S}, d_{I} \geq \bar{D}_{1}$, any positive solution $(S(x), I(x))$ of model (3) satisfies

$$
\underline{C} \leq S(x), \quad I(x) \leq \bar{C} .
$$

Proof. By applying Lemma 5, we have $K\left(1-\left(\mu R_{0} / r\right)\right)<$ $S(x)<K$ for all $x \in \bar{\Omega}$. Let $\phi=D S+d_{I} I>0$ and set $\phi\left(x_{0}\right)=\max _{\bar{\Omega}} \phi$. Then, by applying (i) of Lemma 5 again, we have that

$$
I\left(x_{0}\right) \leq\left(R_{0}-1\right) S\left(x_{0}\right)<\left(R_{0}-1\right) K .
$$

Thus,

$$
\max _{\bar{\Omega}} I \leq d_{I}^{-1} \max _{\bar{\Omega}} \phi \leq K\left(R_{0}-1+D d_{I}^{-1}\right)
$$

Let $c_{1}(x)=\left(1 / d_{S}\right)(r S(1-(S / K))-(\beta S I /(S+I)))$. Then, there exists a positive constant $C=C(\Lambda)$ such that $\left\|c_{1}(x)\right\|_{\infty} \leq C$ provided that $d_{S}>D_{1}$. It follows from Lemma 6 that there exists a positive constant $C^{*}=C^{*}\left(\Lambda, D_{1}\right)$ such that $\max _{\bar{\Omega}} S \leq C^{*} \min _{\bar{\Omega}} S$. On the other hand, $\phi$ satisfies

$$
\begin{gathered}
-\Delta \phi=\frac{(\beta-\mu) S I-\mu I^{2}}{(S+I)\left(D S+d_{I} I\right)} \phi, \quad x \in \Omega, \\
\frac{\partial \phi}{\partial \mathbf{n}}=0, \quad x \in \partial \Omega .
\end{gathered}
$$

Set $c(x)=\left((\beta-\mu) S I-\mu I^{2}\right) /(S+I)\left(D S+d_{I} I\right)$. Then

$$
\|c(x)\|_{\infty} \leq \frac{\beta-\mu}{d_{I}}+\frac{\mu}{d_{I}}=\frac{\beta}{d_{I}} \leq \frac{\beta}{D_{1}} .
$$

It follows from Lemma 6 that there exists a positive constant $C^{*}=C^{*}\left(\Lambda, D_{1}\right)$ such that $\max _{\bar{\Omega}} \phi \leq C^{*} \min _{\bar{\Omega}} \phi$. As a consequence,

$$
\frac{\max _{\bar{\Omega}} I}{\min _{\bar{\Omega}} I}=\frac{\max _{\bar{\Omega}} \phi-D \min _{\bar{\Omega}} S}{\min _{\bar{\Omega}} \phi-D \max _{\bar{\Omega}} S} \leq \frac{C^{*} \min _{\bar{\Omega}} \phi}{\min _{\bar{\Omega}} \varphi-D \max _{\bar{\Omega}} S} \leq C .
$$

Now, it suffices to verify the lower bounds of $I(x)$. We will verify the conclusion by a contradiction argument.

On the contrary, suppose that the conclusion is not true; then there exist sequences $\left\{d_{S, i}\right\}_{i=1}^{\infty},\left\{d_{I, i}\right\}_{i=1}^{\infty}$, and $\left\{D_{i}\right\}_{i=1}^{\infty}$ with $d_{S, i} \geq d, d_{I, i}>D_{1}, D_{i} \geq 0$, and the positive solution $\left(S_{i}, I_{i}\right)$ of model (3) corresponding to $\left(d_{S}, d_{I}, D\right)=\left(d_{S, i}, d_{I, i}, D_{i}\right)$, such that

$$
\min _{\bar{\Omega}} I_{i}(x) \longrightarrow 0 \quad \text { as } i \longrightarrow \infty .
$$


It follows from Lemma 6 that

$$
I_{i}(x) \longrightarrow 0 \quad \text { uniformly on } \bar{\Omega} \text { as } i \longrightarrow \infty \text {. }
$$

$\left(S_{i}, I_{i}\right)$ satisfies

$$
\begin{gathered}
-d_{S, i} \Delta S_{i}=r S_{i}\left(1-\frac{S_{i}}{K}\right)-\frac{\beta S_{i} I_{i}}{S_{i}+I_{i}}, \quad x \in \Omega, \\
-D_{i} \Delta S_{i}-d_{I, i} \Delta I_{i}=\frac{\beta S_{i} I_{i}}{S_{i}+I_{i}}-\mu I_{i}, \quad x \in \Omega, \\
\frac{\partial S_{i}}{\partial \mathbf{n}}=\frac{\partial I_{i}}{\partial \mathbf{n}}=0, \quad x \in \partial \Omega .
\end{gathered}
$$

Integrating by parts, we obtain that, for $i=1,2, \ldots$,

$$
\begin{gathered}
\int_{\Omega} S_{i}\left(r\left(1-\frac{S_{i}}{K}\right)-\frac{\beta I_{i}}{S_{i}+I_{i}}\right) d x=0, \\
\int_{\Omega} I_{i}\left(\frac{\beta S_{i}}{S_{i}+I_{i}}-\mu\right) d x=0 .
\end{gathered}
$$

By the regularity theory for elliptic equations [53], we see that there exist a subsequence of $\left\{\left(S_{i}, I_{i}\right)\right\}_{i}^{\infty}$, which we will still denote by $\left\{\left(S_{i}, I_{i}\right)\right\}_{i}^{\infty}$ and two nonnegative functions $\widetilde{S}, \widetilde{I} \in$ $C^{2}(\Omega)$, such that $\left(S_{i}, I_{i}\right) \rightarrow(\widetilde{S}, \widetilde{I})$ in $\left[C^{2}(\Omega)\right]^{2}$ as $i \rightarrow \infty$. By (39), we have that $\widetilde{I} \equiv 0$. Letting $i \rightarrow \infty$ in (41) we obtain that

$$
\begin{gathered}
\int_{\Omega} \widetilde{S}\left(r-\frac{r}{K} \widetilde{S}-\frac{\beta \widetilde{I}}{\widetilde{S}+\widetilde{I}}\right) d x=0 \\
\int_{\Omega} \widetilde{I}\left(\frac{\beta \widetilde{S}}{\widetilde{S}+\widetilde{I}}-\mu\right) d x=0 .
\end{gathered}
$$

Since $\widetilde{I}=0$, the first equation of (42) becomes $\int_{\Omega} \widetilde{S}(r-$ $(r / K) \widetilde{S}) d x=0$. As $K(1-\beta)<S(x)<K$, we derive a contradiction. This completes the proof.

5.2. Nonexistence of Positive Nonconstant Steady States. This subsection is devoted to the consideration of the nonexistence for the nonconstant positive solutions of model (3), and, in the below results, the diffusion coefficients do play a significant role.

Theorem 8. Assume that $R_{0}>1$. Let $\varepsilon>0$ be an arbitrary constant with $4 d_{S} \varepsilon>D^{2}$ and $d_{I}>\varepsilon$. Then model (3) has no positive nonconstant solution provided that $\left(4 d_{S} \varepsilon-D^{2}\right) \lambda_{1}>$ $4\left(r \varepsilon+\beta^{2}\right)$ and $\left(d_{I}-\varepsilon\right) \lambda_{1}>\mu\left(R_{0}-1\right)+\varepsilon$.

Proof. Let $(S(x), I(x))$ be any positive solution of model (3) and denote $\bar{g}=|\Omega|^{-1} \int_{\Omega} g d x$. Then, multiplying the first equation of model (3) by $(S-\bar{S})$, integrating over $\Omega$, we have that

$$
\begin{aligned}
& d_{S} \int_{\Omega}|\nabla S|^{2} d x \\
& \quad=\int_{\Omega}(S-\bar{S}) S\left(r-\frac{r S}{K}-\frac{\beta I}{S+I}\right) d x \\
& =\int_{\Omega}(S-\bar{S})^{2}\left(r-\frac{r}{K}(S+\bar{S})-\frac{\beta I \bar{I}}{(\bar{S}+\bar{I})(S+I)}\right) d x \\
& \quad-\int_{\Omega} \frac{\beta S \bar{S}}{(\bar{S}+\bar{I})(S+I)}(S-\bar{S})(I-\bar{I}) d x \\
& \quad \leq r \int_{\Omega}(S-\bar{S})^{2}+\beta \int_{\Omega}|S-\bar{S}||I-\bar{I}| d x .
\end{aligned}
$$

In a similar manner, we multiply the second equation in model (3) by $(I-\bar{I})$ to have

$$
\begin{aligned}
\int_{\Omega} & \left(d_{I}|\nabla I|^{2}+D \nabla S \cdot \nabla I\right) d x \\
= & \int_{\Omega}(I-\bar{I})\left(\frac{\beta S I}{S+I}-\mu I\right) P d x \\
= & \int_{\Omega}(I-\bar{I})^{2}\left(-\mu+\frac{\beta \bar{S} S}{(\bar{S}+\bar{I})(S+I)}\right) d x \\
& +\int_{\Omega} \frac{\beta \bar{I} I}{(\bar{S}+\bar{I})(S+I)}(S-\bar{S})(I-\bar{I}) d x \\
\leq & \mu\left(R_{0}-1\right) \int_{\Omega}(I-\bar{I})^{2} d x \\
& +\int_{\Omega} \beta|S-\bar{S}||I-\bar{I}| d x .
\end{aligned}
$$

It follows from (43), (44), and the $\varepsilon$-Young inequality that

$$
\begin{aligned}
& \int_{\Omega}\left(d_{S}|\nabla S|^{2}+d_{I}|\nabla I|^{2}\right) d x \\
& \leq \int_{\Omega}\left(\left(r+\frac{\beta^{2}}{\varepsilon}\right)(S-\bar{S})^{2}+\left(\mu\left(R_{0}-1\right)+\varepsilon\right)(I-\bar{I})^{2}\right) d x \\
& \quad+\int_{\Omega}\left(\frac{D^{2}}{4 \varepsilon}|\nabla S|^{2}+\varepsilon|\nabla I|^{2}\right) d x
\end{aligned}
$$

Thanks to the well-known Poincaré inequality

$$
\lambda_{1} \int_{\Omega}(g-\bar{g})^{2} d x \leq \int_{\Omega}|\nabla(g-\bar{g})|^{2} d x
$$


it follows that

$$
\begin{aligned}
\int_{\Omega} & \left(d_{S}|\nabla S|^{2}+d_{I}|\nabla I|^{2}\right) d x \\
\leq & \int_{\Omega} \frac{4\left(r \varepsilon+\beta^{2}\right)+D^{2} \lambda_{1}}{4 \varepsilon \lambda_{1}}|\nabla S|^{2} d x \\
& +\frac{\mu\left(R_{0}-1\right)+\varepsilon\left(1+\lambda_{1}\right)}{\lambda_{1}} \int_{\Omega}|\nabla I|^{2} d x .
\end{aligned}
$$

Since $4 d_{S} \varepsilon \lambda_{1}>4\left(r \varepsilon+\beta^{2}\right)+D^{2} \lambda_{1}$ and $d_{I} \lambda_{1}>\mu\left(R_{0}-1\right)+$ $\varepsilon\left(1+\lambda_{1}\right)$ from the assumption, one can conclude that $S=\bar{S}$ and $I=\bar{I}$, which asserts our results.

5.3. Existence of Positive Nonconstant Steady States. In this section, we discuss the global existence of nonconstant positive classical solutions to model (3), which guarantees the existence of the stationary patterns $[25,27,29,30]$.

Unless otherwise specified, in this section, we always require that $1<R_{0}<1+(r / \mu)$, which guarantees that model (3) has one positive constant solution $E^{*}$. From now on, let us denote

$$
W=D S+d_{I} I, \quad W^{*}=D S^{*}+d_{I} I^{*} .
$$

We also define

$$
\mathbf{u}=(S, W)
$$

$$
\begin{aligned}
\mathbf{u}^{*}= & \left(S^{*}, W^{*}\right) \\
= & \left(\frac{K}{r}\left(r-\mu\left(R_{0}-1\right)\right),\right. \\
& \left.\quad \frac{K}{r}\left(r-\mu\left(R_{0}-1\right)\right)\left(d_{I}\left(R_{0}-1\right)+D\right)\right) .
\end{aligned}
$$

Let $\mathbf{X}=\left\{\mathbf{u} \in\left[C^{2}(\Omega)\right]^{2} \mid \partial \mathbf{u} / \partial \mathbf{n}=0, x \in \partial \Omega\right\}$ and $\mathbf{X}^{+}=\{\mathbf{u} \epsilon$ $\mathbf{X} \mid S, W>0, x \in \bar{\Omega}\}$. Then we write model (3) in the form

$$
\begin{gathered}
-\Delta \mathbf{u}=\mathbf{G}(\mathbf{u}), \quad x \in \Omega, \\
\frac{\partial \mathbf{u}}{\partial \mathbf{n}}=0, \quad x \in \partial \Omega,
\end{gathered}
$$

where

$$
\mathbf{G}(\mathbf{u})=\left(\begin{array}{c}
\frac{S}{d_{S}}\left(r-\frac{r}{K} S-\frac{\beta(W-D S)}{\left(d_{I}-D\right) S+W}\right) \\
(W-D S)\left(\frac{\beta S}{\left(d_{I}-D\right) S+W}-\frac{\mu}{d_{I}}\right)
\end{array}\right) .
$$

Define a compact operator $\mathscr{F}: \mathbf{X}^{+} \rightarrow \mathbf{X}^{+}$by

$$
\mathscr{F}(\mathbf{u}):=(\mathbf{I}-\Delta)^{-1}\{\mathbf{G}(\mathbf{u})+\mathbf{u}\},
$$

where $(\mathbf{I}-\Delta)^{-1}$ is the inverse operator of $\mathbf{I}-\Delta$ subject to the zero-flux boundary condition. Then $\mathbf{u}$ is a positive solution of model (51) if and only if $\mathbf{u}$ satisfies

$$
(\mathbf{I}-\mathscr{F}) \mathbf{u}=0, \quad x \in \Omega .
$$

To apply the index theory, we investigate the eigenvalue of the problem

$$
-\left(\mathbf{I}-\mathscr{F}_{\mathbf{w}}\left(\mathbf{w}^{*}\right)\right) \Psi=\xi \Psi, \quad \Psi \neq \mathbf{0},
$$

where $\Psi=\left(\Psi_{1}, \Psi_{2}\right)^{T}$ and $\mathscr{F}_{\mathbf{w}}\left(\mathbf{w}^{*}\right)=(\mathbf{I}-\Delta)^{-1}(\mathbf{I}+\mathbf{A})$ with

$$
\mathbf{A}=\left(\begin{array}{cc}
\frac{\mu d_{I} R_{0}^{2}-r d_{I} R_{0}-\mu d_{I}+\mu D}{d_{S} d_{I} R_{0}} & -\frac{\mu}{d_{S} d_{I} R_{0}} \\
\frac{\mu\left(d_{I} R_{0}-d_{I}+D\right)\left(R_{0}-1\right)}{d_{I} R_{0}} & -\frac{\mu\left(R_{0}-1\right)}{d_{I} R_{0}}
\end{array}\right) .
$$

If 0 is not an eigenvalue of (55), by Theorem 2.8.1 in [51], the index of $\mathbf{I}-\mathscr{F}$ at $\mathbf{u}^{*}$ is given by

$$
\operatorname{index}\left(\mathbf{I}-\mathscr{F}, \mathbf{u}^{*}\right)=(-1)^{\gamma} \text {, }
$$

where $\gamma=\sum_{\lambda>0} n_{\lambda}$ and $n_{\lambda}$ is the algebraic multiplicity of the positive eigenvalue $\xi$ of (55).

In fact, after calculation, (55) can be rewritten as

$$
\begin{gathered}
-(\xi+1) \Delta \Psi+(\xi \mathbf{I}-\mathbf{A})=0, \quad x \in \Omega, \\
\frac{\partial \Psi}{\partial \mathbf{n}}=0, \quad x \in \partial \Omega .
\end{gathered}
$$

Observe that (58) has a nontrivial solution if and only if $\operatorname{det}\left(\xi \mathbf{I}+\left(\lambda_{i}+1\right)^{-1}\left(\lambda_{i} \mathbf{I}-\mathbf{A}\right)\right)=0$ for some $\xi \geq 0$ and $i \geq 0$. That is to say, $\xi$ is an eigenvalue of (55), and so (58), if and only if $\xi$ is an eigenvalue of the matrix $\left(\lambda_{i}+1\right)^{-1}\left(\lambda_{i} \mathbf{I}-\mathbf{A}\right)$ for any $i \geq 0$. Therefore, $\mathbf{I}-\mathscr{F}_{\mathbf{u}}\left(\mathbf{u}^{*}\right)$ is invertible if and only if, for any $i \geq 0$ the matrix $\lambda_{i} \mathbf{I}-\mathbf{A}$ is invertible.

Let $m\left(\lambda_{i}\right)$ be the multiplicity of $\lambda_{i}$. For the sake of convenience, we denote

$$
H\left(\lambda_{i}\right)=\operatorname{det}\left(\lambda_{i} \mathbf{I}-\mathbf{A}\right) .
$$

Then if $\lambda_{i} \mathbf{I}-\mathbf{A}$ is invertible for any $i \geq 0$, with the same arguments as in [25], we have

$$
\operatorname{index}\left(\mathscr{F}, \mathbf{u}^{*}\right)=(-1)^{\gamma}, \quad \text { where } \gamma=\sum_{i \geq 0, H\left(\lambda_{i}\right)<0} m\left(\lambda_{i}\right) \text {. }
$$

To compute index $\left(\mathscr{F}, \mathbf{u}^{*}\right)$, we have to consider the sign of $H\left(\lambda_{i}\right)$. A straightforward computation yields

$$
H\left(\lambda_{i}\right)=\lambda_{i}^{2}-\operatorname{trace}(\mathbf{A}) \lambda_{i}+\operatorname{det}(\mathbf{A}),
$$

where

$$
\begin{aligned}
& \operatorname{trace}(\mathbf{A}) \\
& =\frac{\mu d_{I} R_{0}^{2}-\left(\mu d_{S}+r d_{I}\right) R_{0}+\mu d_{S}-\mu d_{I}+\mu D}{d_{S} d_{I} R_{0}}, \\
& \operatorname{det}(\mathbf{A})=\frac{\mu\left(R_{0}-1\right)\left(r-\mu\left(R_{0}-1\right)\right)}{d_{I} R_{0}} .
\end{aligned}
$$

If $\operatorname{trace}(\mathbf{A})^{2}-4 \operatorname{det}(\mathbf{A}) \geq 0$, then $H(\lambda)=0$ has two positive solutions $\lambda^{ \pm}$given by

$$
\lambda^{ \pm}=\frac{1}{2}\left(\operatorname{trace}(\mathbf{A}) \pm \sqrt{\operatorname{trace}(\mathbf{A})^{2}-4 \operatorname{det}(\mathbf{A})}\right) .
$$


Theorem 9. Assume that $\operatorname{trace}(\mathbf{A})^{2}-4 \operatorname{det}(\mathbf{A}) \geq 0$. Then if $\lambda^{-} \in\left(\lambda_{i}, \lambda_{i+1}\right)$ and $\lambda^{+} \in\left(\lambda_{j}, \lambda_{j+1}\right)$ for some $0 \leq i<j$ and $\sum_{k=i+1}^{j} m\left(\lambda_{k}\right)$ is odd, then model (3) has at least one nonconstant solution.

Proof. Suppose that model (3) has no nonconstant positive solution. By Theorem 8 , we can fix $\bar{D}>d_{S}$ such that,

(i) model (3) with diffusion coefficients $\bar{D}, d_{I}$, and $D$ has no nonconstant solutions;

(ii) $H\left(\lambda_{i}\right)>0$ for all $\lambda_{i} \geq 0$.

By virtue of Theorem 7 , there exists a positive constant $C=C(\Lambda, \Omega)$ such that, for $\widehat{d}_{S} \geq d_{S}$, any solution $(S(x), I(x))$ of model (3) with diffusion coefficients $\widehat{d}_{S}, d_{I}$, and $D$ satisfies $C^{-1}<S, I<C$.

Set

$$
\mathscr{M}=\left\{(S, W) \in C(\bar{\Omega}) \times C(\bar{\Omega}): C^{-1}<S, W<C, x \in \bar{\Omega}\right\},
$$

and define

$$
\Phi: \mathscr{M} \times[0,1] \longrightarrow C(\bar{\Omega}) \times C(\bar{\Omega})
$$

by

$$
\Phi(\mathbf{u}, \theta)=(\mathbf{I}-\Delta)^{-1}\{\mathbf{G}(\mathbf{u}, \theta)+\mathbf{u}\}
$$

where

$$
\begin{array}{r}
\mathbf{G}(\mathbf{u}, \theta) \\
=\left(\begin{array}{c}
\left(\theta d_{S}+(1-\theta) \bar{D}\right)^{-1}\left(S\left(r-\frac{r}{K} S-\frac{\beta(W-D S)}{\left(d_{I}-D\right) S+W}\right)\right) \\
(W-D S)\left(\frac{\beta S}{\left(d_{I}-D\right) S+W}-\frac{\mu}{d_{I}}\right)
\end{array}\right) .
\end{array}
$$

It is clear that finding the positive solution of model (51) becomes equivalent to finding the fixed point of $\Phi(\mathbf{u}, 1)$ in $\mathscr{M}$. $\Phi(\mathbf{u}, \theta)$ has no fixed points in $\partial \mathscr{M}$ for all $0 \leq \theta \leq 1$.

Since $\Phi(\mathbf{u}, t)$ is compact, the Leray-Schauder topological degree $\operatorname{deg}(\mathbf{I}-\Phi(\mathbf{u}, \theta), \mathscr{M}, 0)$ is well defined. From the invariance of Leray-Schauder degree at the homotopy, we deduce

$$
\operatorname{deg}(\mathbf{I}-\Phi(\mathbf{u}, 1), \mathscr{M}, 0)=\operatorname{deg}(\mathbf{I}-\Phi(\mathbf{u}, 0), \mathscr{M}, 0) .
$$

Clearly, $\mathbf{I}-\Phi(\mathbf{u}, 1)=\mathbf{I}-\mathscr{F}$. Thus, if model (3) has no other solutions except the constant one $\mathbf{u}^{*}$, we have

$$
\begin{aligned}
& \operatorname{deg}(\mathbf{I}-\Phi(\mathbf{u}, 1), \mathscr{M}, 0) \\
& =\operatorname{index}\left(\mathbf{I}-\mathscr{F}, \mathbf{u}^{*}\right)=(-1)^{\sum_{k=i+1}^{j} m\left(\lambda_{k}\right)}=-1 .
\end{aligned}
$$

On the contrary, by the choice of $\bar{D}$, we have that $\mathbf{u}^{*}$ is the only solution of $\Phi(\mathbf{u}, 0)=0$ and therefore

$$
\operatorname{deg}(\mathbf{I}-\Phi(\mathbf{u}, 0), \mathscr{M}, 0)=\operatorname{index}\left(\mathbf{I}-\mathscr{F}, \mathbf{u}^{*}\right)=(-1)^{0}=1 .
$$

From (68) to (70), we get a contradiction. Therefore, there exists a nonconstant solution of model (3). The proof is completed.

\section{Concluding Remarks}

In this paper, we investigate the effect of cross-diffusion on the disease's dynamics through studying the existence and nonexistence positive constant steady states of a spatial SI epidemic model. The values of this study lie in twofolds. First, we show the local and global stability of the nonnegative steady states, which indicates that the disease reproduction number $R_{0}$ determines whether there is an endemic outbreak or not: the disease free dynamics occurs if $R_{0} \leq 1$ while the unique endemic steady state is globally stable if $\mu<r$ and $1<R_{0}<(r+\mu) / 2 \mu$. Second, we show that even though the unique positive constant steady state (endemic state) is uniformly asymptotically stable for (1), nonconstant positive steady states can exist due to the emergence of crossdiffusion, which demonstrates that stationary patterns can be found as a result of cross-diffusion.

On the other hand, there have been studies of pattern formation in the spatial epidemic model, starting with the pioneering work of Turing [54]. Turing's revolutionary idea was that passive diffusion could interact with the chemical reaction in such a way that even if the reaction by itself has no symmetry-breaking capabilities, diffusion can destabilize the symmetry so that the system with diffusion can have them. Spatial epidemiology with self-diffusion has become a principal scientific discipline aiming at understanding the causes and consequences of spatial heterogeneity in disease transmission. And in the present paper, we prove the existence and nonexistence of the positive nonconstant steady states, which guarantees the existence of the stationary Turing patterns. The numerical results about the Turing patterns for model (1) can be found in [19].

\section{Conflict of Interests}

The authors declare that there is no conflict of interests regarding the publication of this paper.

\section{Acknowledgments}

The authors would like to thank the editors and referees for their helpful comments and suggestions. This research was supported by the National Science Foundation of China (11171357, 61272018, and 61373005) and Zhejiang Provincial Natural Science Foundation (R1110261, and LY12A01014).

\section{References}

[1] W. O. Kermack and A. G. McKendrick, "Contributions to the mathematical theory of epidemics-I," Bulletin of Mathematical Biology, vol. 53, no. 1-2, pp. 33-55, 1991.

[2] Z. Ma, Y. Zhou, and J. Wu, Modeling and Dynamics of Infectious Diseases, Higher Education Press, 2009.

[3] E. E. Holmes, M. A. Lewis, J. E. Banks, and R. R. Veit, "Partial differential equations in ecology: spatial interactions and population dynamics," Ecology, vol. 75, no. 1, pp. 17-29, 1994.

[4] A. Okubo and S. A. Levin, Diffusion and Ecological Problems: Modern Perspectives, vol. 14 of Interdisciplinary Applied Mathematics, Springer, New York, NY, USA, 2nd edition, 2001. 
[5] C. Neuhauser, "Mathematical challenges in spatial ecology," Notices of the American Mathematical Society, vol. 48, no. 11, pp. 1304-1314, 2001.

[6] B. T. Grenfell, O. N. Bjørnstad, and J. Kappey, "Travelling waves and spatial hierarchies in measles epidemics," Nature, vol. 414, no. 6865, pp. 716-723, 2001.

[7] J. D. Murray, Mathematical Biology. II, vol. 18 of Interdisciplinary Applied Mathematics, Springer, New York, NY, USA, 3rd edition, 2003.

[8] L. Rass and J. Radcliffe, Spatial Deterministic Epidemics, vol. 102 of Mathematical Surveys and Monographs, American Mathematical Society, Providence, RI, USA, 2003.

[9] R. S. Cantrell and C. Cosner, Spatial Ecology via ReactionDiffusion Equations, Wiley Series in Mathematical and Computational Biology, John Wiley \& Sons, Chichester, UK, 2003.

[10] A. L. Lloyd and V. A. A. Jansen, "Spatiotemporal dynamics of epidemics: synchrony in metapopulation models," Mathematical Biosciences, vol. 188, pp. 1-16, 2004.

[11] W. M. Van Ballegooijen and M. C. Boerlijst, "Emergent tradeoffs and selection for outbreak frequency in spatial epidemics," Proceedings of the National Academy of Sciences of the United States of America, vol. 101, no. 52, pp. 18246-18250, 2004.

[12] G. Mulone, B. Straughan, and W. Wang, "Stability of epidemic models with evolution," Studies in Applied Mathematics, vol. 118, no. 2, pp. 117-132, 2007.

[13] H. Malchow, S. V. Petrovskii, and E. Venturino, Spatiotemporal Patterns in Ecology Andepidemiology-Theory, Models, and Simulation, Mathematical and Computational Biology Series, Chapman \& Hall/CRC, Boca Raton, Fla, USA, 2008.

[14] R. K. Upadhyay, N. Kumari, and V. S. H. Rao, "Modeling the spread of bird flu and predicting outbreak diversity," Nonlinear Analysis: Real World Applications, vol. 9, no. 4, pp. 1638-1648, 2008.

[15] K. Wang, W. Wang, and S. Song, "Dynamics of an HBV model with diffusion and delay," Journal of Theoretical Biology, vol. 253, no. 1, pp. 36-44, 2008.

[16] G.-Q. Sun, Z. Jin, Q.-X. Liu, and L. Li, "Spatial pattern in an epidemic system with cross-diffusion of the susceptible," Journal of Biological Systems, vol. 17, no. 1, pp. 141-152, 2009.

[17] M. Bendahmane and M. Saad, "Mathematical analysis and pattern formation for a partial immune system modeling the spread of an epidemic disease," Acta Applicandae Mathematicae, vol. 115, no. 1, pp. 17-42, 2011.

[18] Y. Cai and W. Wang, "Spatiotemporal dynamics of a reactiondiffusion epidemic model with nonlinear incidence rate," Journal of Statistical Mechanics: Theory and Experiment, vol. 2011, no. 2, Article ID P02025, 2011.

[19] W. Wang, Y. Lin, H. Wang, H. Liu, and Y. Tan, "Pattern selection in an epidemic model with self and cross diffusion," Journal of Biological Systems, vol. 19, no. 1, pp. 19-31, 2011.

[20] W. Wang, Y. Cai, M. Wu, K. Wang, and Z. Li, "Complex dynamics of a reaction-diffusion epidemic model," Nonlinear Analysis: Real World Applications, vol. 13, no. 5, pp. 2240-2258, 2012.

[21] Y. Cai, W. Liu, Y. Wang, and W. Wang, "Complex dynamics of a diffusive epidemic model with strong Allee effect," Nonlinear Analysis: Real World Applications, vol. 14, no. 4, pp. 1907-1920, 2013.

[22] Y. Lou and W.-M. Ni, "Diffusion, self-diffusion and crossdiffusion," Journal of Differential Equations, vol. 131, no. 1, pp. 79-131, 1996.
[23] E. H. Kerner, "Further considerations on the statistical mechanics of biological associations," The Bulletin of Mathematical Biophysics, vol. 21, no. 2, pp. 217-255, 1959.

[24] N. Shigesada, K. Kawasaki, and E. Teramoto, "Spatial segregation of interacting species," Journal of Theoretical Biology, vol. 79, no. 1, pp. 83-99, 1979.

[25] M. Wang, "Non-constant positive steady states of the Sel'kov model," Journal of Differential Equations, vol. 190, no. 2, pp. $600-$ 620, 2003.

[26] P. Y. H. Pang and M. Wang, "Strategy and stationary pattern in a three-species predator-prey model," Journal of Differential Equations, vol. 200, no. 2, pp. 245-273, 2004.

[27] M. Wang, "Stationary patterns of strongly coupled preypredator models," Journal of Mathematical Analysis and Applications, vol. 292, no. 2, pp. 484-505, 2004.

[28] M. Wang, "Stationary patterns caused by cross-diffusion for a three-species prey-predator model," Computers \& Mathematics with Applications, vol. 52, no. 5, pp. 707-720, 2006.

[29] R. Peng, J. Shi, and M. Wang, "Stationary pattern of a ratiodependent food chain model with diffusion," SIAM Journal on Applied Mathematics, vol. 67, no. 5, pp. 1479-1503, 2007.

[30] R. Peng, J. Shi, and M. Wang, "On stationary patterns of a reaction-diffusion model with autocatalysis and saturation law," Nonlinearity, vol. 21, no. 7, pp. 1471-1488, 2008.

[31] R. Peng and J. Shi, "Non-existence of non-constant positive steady states of two Holling type-II predator-prey systems: strong interaction case," Journal of Differential Equations, vol. 247, no. 3, pp. 866-886, 2009.

[32] F. Yi, J. Wei, and J. Shi, "Bifurcation and spatiotemporal patterns in a homogeneous diffusive predator-prey system," Journal of Differential Equations, vol. 246, no. 5, pp. 1944-1977, 2009.

[33] B. Li and M. Wang, "Stationary patterns of the stage-structured predator-prey model with diffusion and cross-diffusion," Mathematical and Computer Modelling, vol. 54, no. 5-6, pp. 13801393, 2011.

[34] S. A. Levin and L. A. Segel, "Pattern generation in space and aspect," SIAM Review, vol. 27, no. 1, pp. 45-67, 1985.

[35] M. Barthélemy, A. Barrat, R. Pastor-Satorras, and A. Vespignani, "Dynamical patterns of epidemic outbreaks in complex heterogeneous networks," Journal of Theoretical Biology, vol. 235, no. 2, pp. 275-288, 2005.

[36] V. Colizza and A. Vespignani, "Epidemic modeling in metapopulation systems with heterogeneous coupling pattern: theory and simulations," Journal of Theoretical Biology, vol. 251, no. 3, pp. 450-467, 2008.

[37] O. G. Jepps, C. Bianca, and L. Rondoni, "Onset of diffusive behavior in confined transport systems," Chaos, vol. 18, no. 1, Article ID 013127, 2008.

[38] J. R. Cannon and D. J. Galiffa, "An epidemiology model suggested by yellow fever," Mathematical Methods in the Applied Sciences, vol. 35, no. 2, pp. 196-206, 2012.

[39] K. P. Das and J. Chattopadhyay, "Role of environmental disturbance in an eco-epidemiological model with disease from external source," Mathematical Methods in the Applied Sciences, vol. 35, no. 6, pp. 659-675, 2012.

[40] C. Bianca, "Existence of stationary solutions in kinetic models with gaussian thermostats," Mathematical Methods in the Applied Sciences, vol. 36, no. 13, pp. 1768-1775, 2013.

[41] K. I. Kim, Z. Lin, and Q. Zhang, "An SIR epidemic model with free boundary," Nonlinear Analysis: Real World Applications, vol. 14, no. 5, pp. 1992-2001, 2013. 
[42] Z. Liu, "Dynamics of positive solutions to SIR and SEIR epidemic models with saturated incidence rates," Nonlinear Analysis: Real World Applications, vol. 14, no. 3, pp. 1286-1299, 2013.

[43] M. Qiao, A. Liu, and U. Foryś, "Qualitative analysis of the SICR epidemic model with impulsive vaccinations," Mathematical Methods in the Applied Sciences, vol. 36, no. 6, pp. 695-706, 2013.

[44] L. Wang, Z. Teng, and H. Jiang, "Global attractivity of a discrete SIRS epidemic model with standard incidence rate," Mathematical Methods in the Applied Sciences, vol. 36, no. 5, pp. 601-619, 2013.

[45] S. Wang, W. Liu, Z. Guo, and W. Wang, "Traveling wave solutions in a reaction-diffusion epidemic model," Abstract and Applied Analysis, vol. 2013, Article ID 216913, 13 pages, 2013.

[46] A. Pazy, Semigroups of Linear Operators and Applications to Partial Differential Equations, vol. 44 of Applied Mathematical Sciences, Springer, New York, NY, USA, 1983.

[47] M. Kirane, "Global bounds and asymptotics for a system of reaction-diffusion equations," Journal of Mathematical Analysis and Applications, vol. 138, no. 2, pp. 328-342, 1989.

[48] D. Henry, Geometric Theory of Semilinear Parabolic Equations, vol. 840 of Lecture Notes in Mathematics, Springer, Berlin, Germany, 1981.

[49] L. Melkemi, A. Z. Mokrane, and A. Youkana, "On the uniform boundedness of the solutions of systems of reaction-diffusion equations," Electronic Journal of Qualitative Theory of Differential Equations, no. 24, pp. 1-10, 2005.

[50] E. H. Daddiouaissa, "Existence of global solutions for a system of reaction-diffusion equations having a triangular matrix," Electronic Journal of Differential Equations, vol. 2008, no. 141, pp. 1-9, 2008.

[51] L. Nirenberg, Topics in Nonlinear Functional Analysis, vol. 6, AMS Bookstore, 2001.

[52] C.-S. Lin, W.-M. Ni, and I. Takagi, "Large amplitude stationary solutions to a chemotaxis system," Journal of Differential Equations, vol. 72, no. 1, pp. 1-27, 1988.

[53] D. Gilbarg and N. S. Trudinger, Elliptic Partial Differential Equations of Second Order, vol. 224, Springer, Berlin, Germany, 2nd edition, 1983.

[54] A. M. Turing, "The chemical basis of morphogenesis," Philosophical Transactions of The Royal Society of London B, vol. 237, no. 641, pp. 37-72, 1952. 


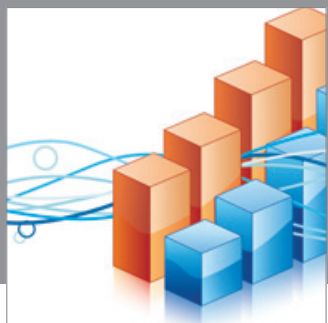

Advances in

Operations Research

mansans

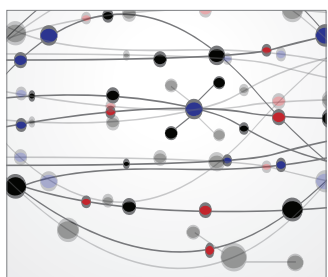

The Scientific World Journal
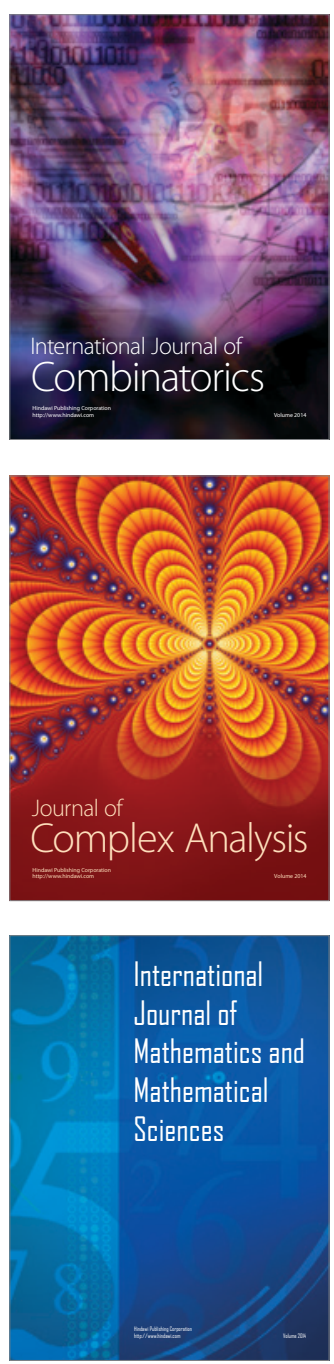
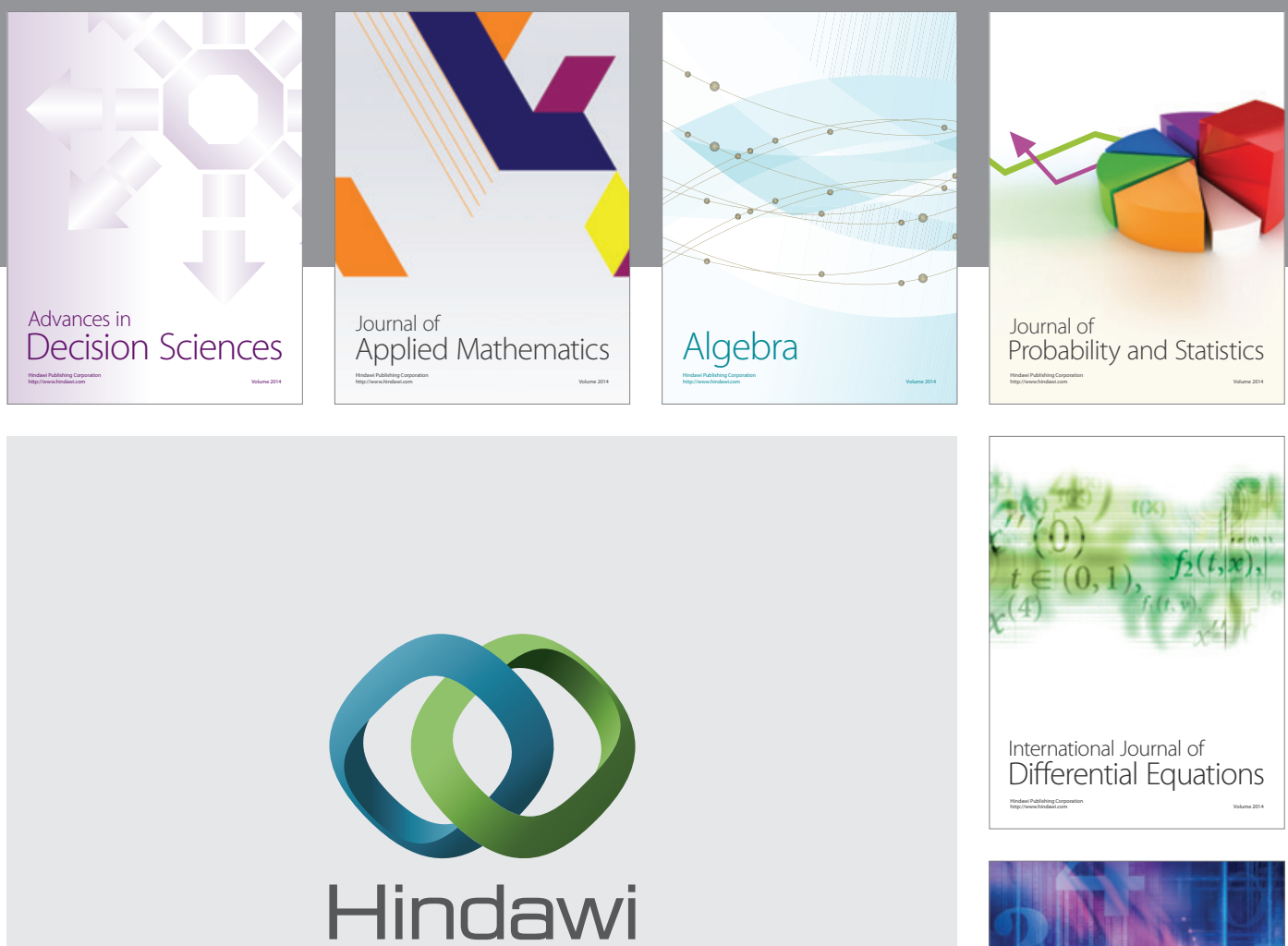

Submit your manuscripts at http://www.hindawi.com
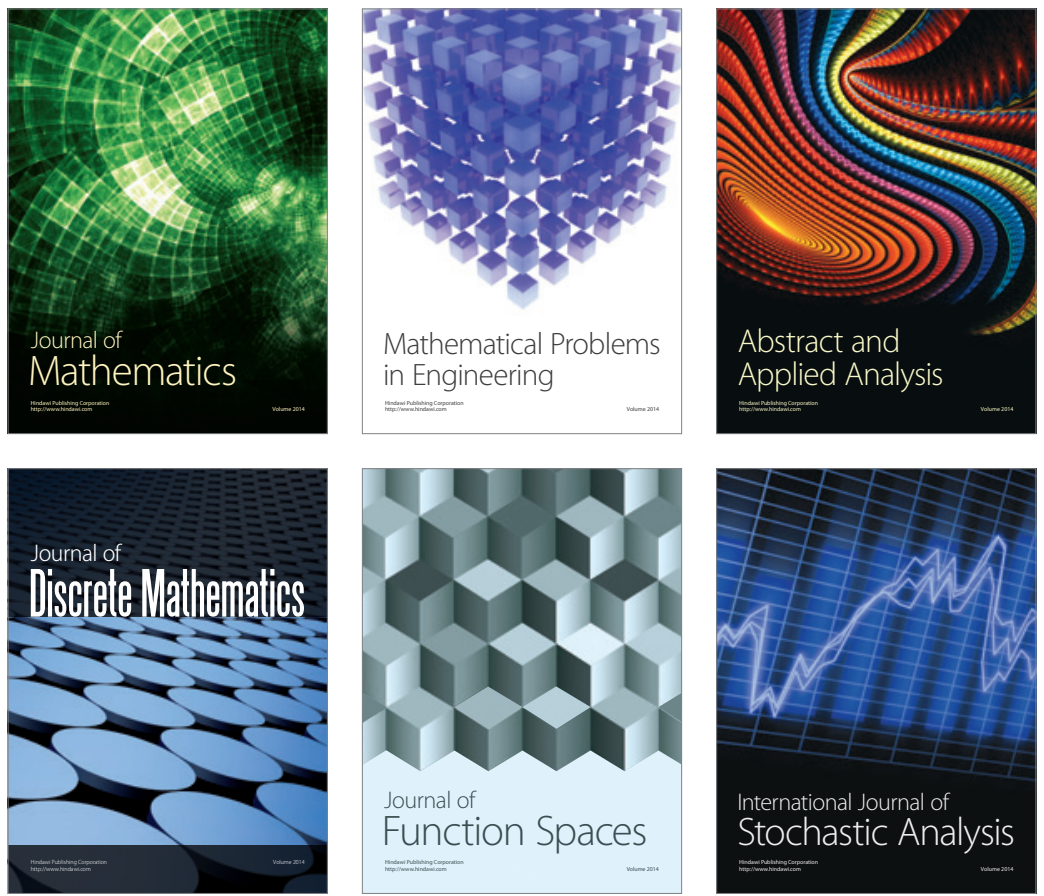

Journal of

Function Spaces

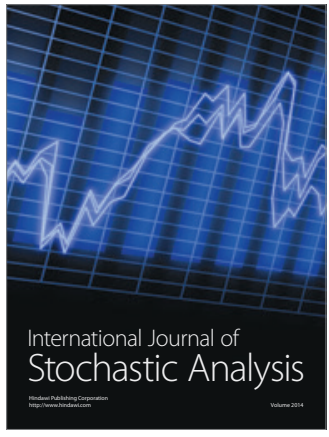

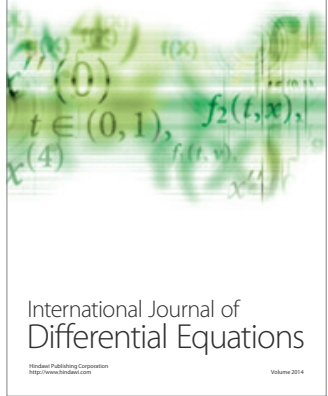
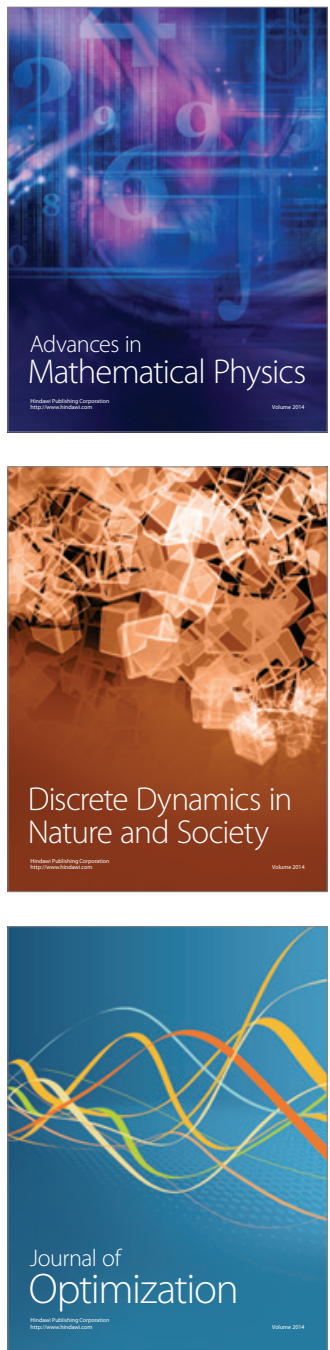